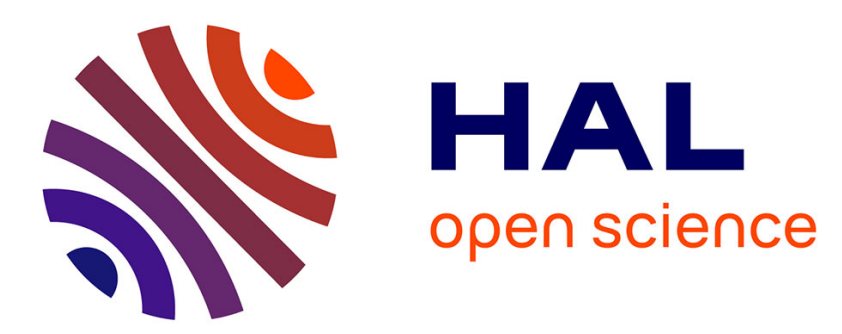

\title{
Adaptation des systèmes de contrôle en temps de crise. Perceptions et réactions des commerciaux
}

\author{
Madeleine Besson, Laure Lavorata
}

\section{To cite this version:}

Madeleine Besson, Laure Lavorata. Adaptation des systèmes de contrôle en temps de crise. Perceptions et réactions des commerciaux. Revue Française de Gestion, 2013, 7 (236), pp.15-31. 10.3166/rfg.236.15-31 . hal-01123789

\section{HAL Id: hal-01123789 \\ https://hal.science/hal-01123789}

Submitted on 2 Mar 2016

HAL is a multi-disciplinary open access archive for the deposit and dissemination of scientific research documents, whether they are published or not. The documents may come from teaching and research institutions in France or abroad, or from public or private research centers.
L'archive ouverte pluridisciplinaire HAL, est destinée au dépôt et à la diffusion de documents scientifiques de niveau recherche, publiés ou non, émanant des établissements d'enseignement et de recherche français ou étrangers, des laboratoires publics ou privés. 


\title{
Adaptation des systèmes de contrôle en temps de crise :
}

\author{
perceptions et réactions des commerciaux
}

\author{
Madeleine BESSON, Institut Mines Télécom / Télécom Ecole de management \\ Laure LAVORATA, Université Paris Est / I.R.G.
}

\section{Résumé}

Cet article se propose d'étudier l'impact de la crise économique sur les organisations commerciales, et les modalités d'adaptation des systèmes de contrôle face à ce contexte inédit. Les résultats issus d'une approche qualitative menée auprès de commerciaux montrent que ces derniers perçoivent un renforcement de la pression par le management dans l'entreprise qui se traduit par un reporting accru (1) et que le comportement des clients contribue à influencer les systèmes de contrôle à travers l'impact sur les commerciaux (2). Le troisième résultat réside dans l'importance du contrôle informel (self control) développé par le commercial pour s'adapter au client.

Mots-clefs : management commercial, systèmes de contrôle, crise, étude qualitative, commerciaux, comportement des clients

\section{Adaptive behavior of salespeople and adaptive sales force control due to the economic} crisis: results of an exploratory study.

\begin{abstract}
This article aims at analyzing the impact of the economic crisis on commercial organizations and how they have adapted their systems of control. The results of a qualitative approach among 58 salespeople show that (1) salespeople perceive an increased pressure in the daily management and suggest that (2) customers' behavior can influence salespeople's behavior and indirectly impact the sales force control system. The third result is the importance of selfcontrol developed by the salesperson to adapt to the customer.
\end{abstract}

Key-words: sales management, sales force control, economic crisis, qualitative study, salespeople, customers' behaviors 
Il ne fait plus de doute aujourd'hui que la crise économique actuelle est la plus importante des crises auxquelles ont été confrontées les sociétés occidentales depuis 1929. L'enchaînement des événements qui ont été relayés dans les media sous l'appellation de crise financière (2008), de crise économique (2009) puis de crise de la dette souveraine (2011) forme un environnement objectivement incertain. Cet environnement, caractérisé par des périodes de récession économique a représenté de nouveaux défis en termes de gestion des entreprises dans la période récente, à même de révéler dans ces organisations une crise organisationnelle de forte ampleur. Récemment, six experts de la gestion ont été interrogés afin d'identifier ce que la crise avait remis en cause dans chacune de leur discipline (Revue Française de Gestion, 2009) : l'une des interrogations de ces experts porte sur le rôle du «pilotage par le chiffre» ainsi que de la vision mécaniste qui domineraient les pratiques de gestion » dans la crise (Barthelemy et Doublet, 2009, p. 14).

A ce titre, il nous a semblé intéressant d'analyser le management d'une entité opérationnelle dans ce contexte particulier, «où l'organisation doit s'organiser pour faire face à la nouvelle situation» (Weick, 2001 p. 27). Notre questionnement porte plus spécifiquement sur la manière dont les entreprises ont adapté leur système de contrôle dans un contexte de crise et les réactions que les commerciaux ont développées face aux conséquences en termes de management commercial. En conséquence, notre recherche s'efforce de répondre aux questions suivantes :

- Les systèmes de contrôle sont-ils modifiés par la crise et comment les commerciaux perçoivent-ils cette évolution? Le pilotage de la force de vente utilisé en temps habituel fait-il l'objet d'adaptations et comment celles-ci sont-elles perçues ?

- Comment les commerciaux, en contact avec les clients, perçoivent-ils et répondent-ils à la crise ? Des dérives éthiques sont-elles perceptibles ? 
Il existe peu de recherches empiriques sur l'impact d'une crise sur les systèmes de pilotage des forces de vente (Bergadaà, 2000). Pourtant, du fait de leur position intermédiaire entre les clients et l'organisation, les commerciaux sont, plus que d'autres, soumis aux mutations de l'environnement, et doivent faire face à l'évolution du comportement des clients. Des entretiens réalisés auprès de commerciaux ont permis d'analyser de manière plus précise leur perception de la crise et des ajustements apportés par les organisations commerciales dans cet environnement. Sur le plan académique, notre recherche permet d'enrichir les travaux antérieurs sur le pilotage des forces de vente. Elle apporte en particulier une vérification empirique de certaines propositions proposées récemment par Darmon et Martin (2011). Nous suggérons ainsi de compléter le cadre conceptuel de Jaworski (1988) en intégrant une nouvelle partie prenante que sont les clients de l'entreprise. Sur le plan managérial, cette recherche peut apporter un nouvel éclairage sur l'influence de l'environnement externe et plus particulièrement celui de la crise sur les systèmes de contrôle des forces de vente.

Nous détaillons ci-après le cadre théorique (I), la méthodologie (II), les enseignements tirés de cette étude (III) ; finalement, nous proposons un cadre conceptuel issu de la littérature et des résultats de nos analyses (IV).

\section{I - CADRE THEORIQUE}

Darmon (2001) propose une vision systémique du management de la force de vente intégrant deux niveaux. Le management stratégique de la force de vente consiste à «fixer les objectifs et l'organisation commerciale »; au niveau opérationnel, le management commercial consiste à «piloter l'action commerciale afin d'atteindre ces objectifs». S'il est peu probable que l'évolution de l'environnement économique puisse amener l'entreprise à modifier 
radicalement l'organisation commerciale et ses objectifs en l'espace de quelques mois, on peut supposer que l'organisation tentera néanmoins de s'adapter au niveau opérationnel.

Dans cette partie théorique, nous définirons tout d'abord le concept de crise avant de présenter les caractéristiques des systèmes de contrôle des forces de vente, puis les conséquences des modifications de l'environnement de l'entreprise sur le pilotage et le management de ses équipes de vente.

\section{Définition de la crise}

Etudié depuis les années 1960 dans le champ du management, le concept de crise continue à faire débat. Certains auteurs pointent le manque de consensus sur le concept même de crise (Kouzmin, 2008), et les difficultés des chercheurs à s'accorder sur trois éléments importants (Adrot, 2010) :

- Le caractère exogène ou endogène de la crise : du point de vue de la littérature en management, la crise peut être vue comme un événement rare et non anticipé ; d'un autre côté, cet événement révèle le plus souvent des dysfonctionnements ou faiblesses internes à l'organisation,

- La distinction entre difficultés des organisations et situations de crise : faire face à des difficultés fait partie de la vie de l'organisation. Pour les chercheurs en management, la crise se distingue par son ampleur, en particulier en termes de coûts sociaux liés à la crise

- Le caractère objectif ou subjectif de la crise : la crise peut être qualifiée avec des indicateurs objectifs tels des coûts économiques, mais également analysée comme une «expérience personnelle ( Mitroff et al., 1996).

Même si des divergences de vue se font jour parmi les auteurs, il semble que l'on puisse caractériser la crise comme une situation dans laquelle «de nombreux membres de 
l'organisation (social system) se trouvent privés des conditions de vie qu'ils en attendent» (Barton, 1969, cité par Quarantelli and Dynes, 1977).

La littérature consacrée au management de crise par les entreprises a donné lieu à des préconisations portant sur la gestion de la crise en interne. Une place importante est ainsi faite à la question de la communication, au sein de l'organisation comme auprès des parties prenantes (Coombs, 2007) et deux perspectives se distinguent: le premier courant de recherche insiste sur l'importance d'une information claire, convergente et décodée de façon non ambiguë par les acteurs. La seconde approche suggère que face à une situation de crise les acteurs doivent partager une vision commune afin de pouvoir ensuite improviser et développer des stratégies cohérentes (Roux-Dufort et Vidaillet, 2003).

Pour notre part, nous considérons que la crise économique qui sévit en Europe depuis 2009 est à la fois objective (avérée par des indicateurs tels que des périodes de récession économique ou des licenciements), et vécue de façon subjective et potentiellement différente par les acteurs de différents secteurs. En conséquence, dans le cadre de cette étude, nous nous centrerons sur le caractère exogène de la crise, et les conséquences qu'elle peut avoir en particulier sur les systèmes de contrôle en interne.

\section{Systèmes de contrôle des forces de vente}

L'article d'Anderson et Olivier marque le début d'un important courant de recherche sur les systèmes de contrôle des forces de vente dont ils proposent la définition suivante : «un système de procédures mises en place par l'organisation pour piloter, diriger, évaluer et rétribuer ses collaborateurs» (Anderson et Olivier, 1987, p. 76). A la même époque, Jaworski propose de définir le contrôle comme une «tentative des managers d'influencer le comportement et les activités des collaborateurs (...) pour atteindre les objectifs souhaités» (1988, p.58). 
Fondées sur les mêmes théories organisationnelles, entre autres sur les travaux d'Ouchi (1980), les deux propositions théoriques ont conduit les auteurs et leurs successeurs à développer des mesures différentes du contrôle des forces de vente. En effet, Jaworski (1988) suggère de distinguer les contrôles formels des contrôles informels : les premiers sont de nature écrites, initiés par le management; les seconds incluent des mécanismes sociaux de contrôle (self-control, contrôle social du groupe ou contrôle culturel), mécanismes par définition non formalisés et à l'initiative de l'individu et non du management.

A l'inverse, Anderson et Oliver (1987) suggèrent que deux philosophies du pilotage de la force de vente s'opposent et stigmatisent, l'une le contrôle exercé sur le comportement du vendeur, l'autre, le contrôle de ses résultats ; dans cette vision, la philosophie du contrôle et les éléments de contrôle sur lesquels elle s'appuie sont le fait exclusif du management. Entre ces deux pôles (contrôle par les comportements versus contrôle par les résultats) s'établissent des positions médianes, dits systèmes de contrôle «hybrides »(Olivier et Anderson, 1995), des systèmes très majoritaires en France à la fin des années 1990 (Rouziès et Besson, 1998). Les travaux empiriques qui se réfèrent au cadre développé par Anderson et Oliver (1987) proposent des mesures composites qui, selon les auteurs, incluent entre quatre dimensions (Krafft, 1999) et six dimensions (Oliver et Anderson, 1994). Au sein de ce courant de recherche, les chercheurs se sont essentiellement attachés à analyser les impacts du choix de différents systèmes de contrôle par les entreprises sur le comportement des vendeurs et la performance de l'entreprise.

Sur la base du cadre théorique développé en 1988, Jaworski et al. (1993) proposent de distinguer quatre types de systèmes de contrôle (contrôle fort, contrôle social, bureaucratie ou faible niveau de contrôle), et analysent les prédicteurs et les conséquences de ces systèmes ; un système de contrôle «fort» (niveaux de contrôle formel et informel élevés) semble 
prédire une meilleure performance, résultat confirmé par l'étude de Cravens et al. (2004). Challagalla et Shervani (1996) complètent la mesure du système de contrôle en incluant la dimension d'information de ce système et sa capacité de renforcement des attitudes par les récompenses. Alors que la perspective traditionnelle du contrôle met l'accent sur les effets dysfonctionnels des contrôles formels sur les salariés, Ramaswami (1996) suggère que la considération que les salariés perçoivent de la part de leur supérieur hiérarchique est en mesure de réduire les comportements dysfonctionnels dus aux contrôles formalisés.

A l'issue d'une revue de la littérature consacrée aux systèmes de contrôle des forces de vente, Baldauf et al. (2005) concluent à l'impossibilité à ce stade d'unifier les deux visions des systèmes de contrôle des forces de ventes, et appellent à un effort de théorisation complémentaire pour proposer une vision unifiée de ce concept.

Récemment, Darmon et Martin (2011) ont proposé un nouveau cadre intégrateur pour analyser les systèmes de contrôle des forces de vente. Ce cadre conceptuel prend en compte la nature dynamique des systèmes de contrôle et introduit les concepts d'intensité de l'usage qui est fait des différents outils de contrôle, et de la «rigueur du renforcement » du contrôle exercé par le management.

En synthèse, le système de contrôle des forces de vente apparaît comme «un processus d'influence que le management utilise pour inciter les vendeurs à travailler à la réalisation des objectifs de l'entreprise à court et à long terme » (Darmon et Martin, 2011, p.301). Le management de proximité conserve la possibilité de palier des dysfonctionnements des contrôles formels mis en place par l'entreprise, en augmentant ou allégeant l'usage des outils de contrôle, et accroissant ou non le soutien qu'ils prodiguent à leurs équipes. Si la crise nécessite de partager une «vision commune » au sein de l'équipe commerciale, comment cela 
se répercute-t-il à la fois en termes de système de contrôles formels et sur le plan du management au quotidien?

\section{Impact des facteurs externes sur le pilotage des organisations commerciales}

Les résultats des études empiriques consacrées aux systèmes de contrôle des forces de vente portent très majoritairement sur les impacts du choix de tel ou tel système de contrôle. Quelques auteurs ont néanmoins tenté de comprendre quels en étaient les antécédents.

Sur le plan théorique, Jaworski (1988) a proposé un cadre de compréhension des modalités de contrôle des activités marketing. L'auteur propose d'identifier différents facteurs explicatifs du système de contrôle adopté par l'entreprise dans l'environnement interne et externe de la firme, et suggère que le contexte dans lequel se meut l'entreprise a un rôle prépondérant dans la détermination des systèmes de contrôle.

$\mathrm{Au}$ terme d'une étude menée en Allemagne, Krafft conclut que le choix du système de contrôle dépend bien plus des conditions du marché que des caractéristiques de la force de vente (1999, p.129). Entre autres, des entreprises travaillant sur des marchés à forte volatilité ont tendance à mettre en place des systèmes de contrôle des comportements plutôt qu'un contrôle basé sur les résultats. Cependant, qu'en est-il lorsque l'incertitude s'invite brutalement sur l'ensemble des marchés ?

Cravens et al. (1993) ont analysé le rôle joué par les périodes hautes et basses des cycles économiques aux Etats-Unis et indiquent que «lors de périodes défavorables sur le plan économique [...] il est important de renforcer le niveau de motivation intrinsèque des commerciaux » (p 56). Si l'on exclut ces travaux, les études empiriques relatives à l'impact des facteurs environnementaux sur les systèmes de pilotage des organisations commerciales restent très parcellaires. Sans doute faut-il voir dans la difficulté à donner des réponses empiriques à cette question de recherche une conséquence des difficultés auxquelles se 
confrontent les chercheurs qui cherchent à mesurer de façon quantitative les éléments de l'environnement externe de l'entreprise. Dans cette étude, nous nous proposons de pallier cette difficulté en utilisant une méthode qualitative permettant de prendre en compte le contexte et de l'analyser de manière plus fine.

\section{METHODOLOGIE}

Deux études qualitatives ont été menées successivement sous forme d'entretiens semidirectifs (encadré 1). Nous avons choisi de réaliser une première étude dans le secteur bancaire afin d'identifier l'impact immédiat de la crise sur le management commercial dans ce secteur, le plus directement touché par la crise financière en 2008/2009. Nous nous sommes ensuite interrogés sur l'impact que celle-ci pouvait avoir eu dans d'autres secteurs comme l'immobilier, l'automobile et nous avons alors choisi de comparer les effets de la crise économique sur des secteurs différents un an plus tard. Ces études avaient pour objectif de déterminer si les systèmes de contrôle pouvaient effectivement être différents dans un secteur plus fortement et directement touché par la crise et de montrer la réalité perçue par des commerciaux.

Nous aurions pu utiliser le principe des études longitudinales qui, selon Greene (2003 p.283), «consistent à mesurer sur plusieurs périodes les mêmes objets avec les mêmes variables afin de comprendre leur évolution au cours du temps ». Cependant ces études sont surtout utilisées sous la forme de tests avant/ après et dans notre étude, la crise étant survenue rapidement, il était difficile de revenir sur les pratiques antérieures. En revanche, il nous a semblé plus intéressant de comparer l'impact sur des secteurs différents afin de déterminer si l'évolution des pratiques commerciales était identique ou bien s'il y avait des différences. Des auteurs ont mis en avant l'intérêt des études comparatives qui, si elles sont d'abord utilisées au niveau 
international, permettent cependant de faire des comparaisons entre secteurs : ainsi Boddewyn (1966, p. 149) souligne que «l'approche comparative permet de détecter, d'identifier, de classifier, de mesurer et d'interpréter de manière systématique des similitudes et des différences à propos d'un phénomène » et que c'est en cela qu'elle est pertinente dans le domaine du marketing.

\section{Encadré 1. Méthodologie de la recherche}

La première étude a été réalisée en mars 2009, 6 mois après la faillite de Lehman Brother aux Etats-Unis. Douze entretiens ont été conduits dans le secteur bancaire auprès de 2 établissements. Le choix de la banque a été privilégié du fait de l'impact important qu'a eu la crise des subprimes sur ce secteur en 2008/2009. Les conseillers bancaires interrogés se répartissaient comme suit : 5 hommes et 7 femmes dont la moyenne d'âge est de 33 ans et l'ancienneté dans la fonction commerciale de 5 ans.

L'étude a été élargie à de nouveaux secteurs en 2010 afin d'analyser comment l'évolution de la crise économique avait été prise en compte dans les entreprises et d'identifier une éventuelle contingence sectorielle dans les réactions des organisations commerciales face à la crise. Conduite en avril 2010, La seconde étude, a permis d'interviewer 46 commerciaux, dans des secteurs divers comme l'immobilier, l'automobile ou l'équipement de la maison et auprès d'entreprises qui ont accepté de participer à cette étude (moyenne d'âge : 37 ans ; ancienneté moyenne : 13 ans ; femmes : 30\%). Dans cette seconde étude, les commerciaux travaillant avec une clientèle de particuliers (B2C) représentent les deux tiers des interviewés. Le même guide d'entretien a été utilisé afin d'identifier les divergences ou similitudes selon les secteurs (Annexe 1).

Au total, ce sont donc 58 entretiens semi-directifs qui ont été conduits auprès de commerciaux (Annexe 2). Une analyse de contenu thématique a été réalisée sur la base d'une retranscription 
intégrale des entretiens. Dans la mesure où notre objectif était de mettre en évidence les similitudes et les différences dans les opinions des commerciaux - et non d'identifier une typologie de vendeurs -, nous avons opté pour une analyse de contenu horizontale. Le choix d'une analyse interprétative a été privilégié car, bien que le logiciel ALCESTE soit intéressant pour mettre en évidence des similitudes et des occurrences, il n'est pas toujours pertinent lorsque l'on cherche à mettre en évidence des différences. Par ailleurs comme le soulignent Kalampalikis et Moscovici (2005), lorsque le corpus est abondant et redondant, les occurrences ou les thématiques sont difficilement interprétables : «de quoi parle-t-on lorsque l'on répète souvent les mots » (p.20).

\section{III - PERCEPTION DE L'IMPACT DE LA CRISE PAR LES COMMERCIAUX}

Dans cette partie, nous allons développer les trois principaux résultats de notre étude: les évolutions des systèmes de contrôle (1), l'impact de la crise sur le comportement du client (2) et les réactions des commerciaux face à ces nouveaux comportements (3).

\section{Quels systèmes de contrôle face à la crise ?}

Dès les débuts de la crise, et par la suite, quels que soient les secteurs, les commerciaux interrogés rapportent de modifications sensibles du management dans leur organisation : la pression et les contrôles opérés par le management sont partout renforcés (a), alors que les initiatives pour accompagner et remotiver les vendeurs semblent beaucoup plus rares (b).

\section{a. Pression et contrôle accrus}

En temps de crise, l'augmentation de la pression sur les commerciaux est générale. Ainsi, dans le secteur bancaire, la perception exprimée par les conseillers lors des entretiens laisse percevoir une pression exercée au quotidien par le management dans l'agence : «[Au fil des 
années], on est passé d'objectifs de conseil à des objectifs de ventes », mais aussi d'une perception croissante de pression sur le chiffre, et de «flicage» (sic) :

«Aujourd'hui c'est vraiment du chiffre, du chiffre et du chiffre [...] on est vérifié, regardé, scruté. »

Alors que la structure de rémunération du secteur bancaire est en ligne avec un contrôle des comportements (forte part du salaire fixe), le management de proximité impose une pression accrue sur les résultats. A l'entretien annuel d'évaluation classique s'est ajouté au fil du temps, un entretien mensuel et parfois même un suivi quotidien des résultats par les responsables des agences bancaires. «Le nombre de ventes est suivi de façon quotidienne [...] il y a plus de pression, plus de contrôle».

Cette pression est ressentie de la même manière dans les autres secteurs puisque les commerciaux déclarent: «on bouffe du client »; «on doit être au top en permanence ». Face à un environnement plus concurrentiel, le management commercial instaure également de nouveaux contrôles sur les commerciaux : «Il y a plus de vérification des comptes clients » ou de «suivi des encours clients ». Dans la banque, le management accentue les procédures et le travail administratif : "l'accent a été mis sur le respect des normes, des procédures de plus en plus strictes »; «les comparaisons chiffrées sont de plus en plus présentes »; «plus de contrôle; les objectifs sont plus ambitieux; il y a plus d'administratif ».

Au-delà même de la pression sur les résultats et des contrôles renforcés, des commerciaux mentionnent la révision à la hausse de leurs objectifs de vente et ce «dans un marché en recul!».

$\underline{\text { b. Quelques initiatives pour remotiver les commerciaux dans certaines entreprises }}$

A l'inverse, quelques initiatives laissent penser que certaines organisations ont perçu la nécessité d'apporter un support à leur force de vente et de les remotiver dans une période 
difficile. Ainsi, dans un contexte de baisse du chiffre d'affaires, certaines entreprises ont choisi de revoir à la baisse les objectifs de vente assignés à leurs commerciaux afin «d'éviter une démotivation des commerciaux». On note également un accompagnement managérial accru dans certaines entreprises soucieuses de maintenir la motivation de leurs commerciaux. Un support est parfois offert aux commerciaux terrain ; ainsi, un acteur de l'immobilier a-t-il opté pour le «recrutement d'une secrétaire afin que les commerciaux se concentrent davantage sur la vente».

Face à la détérioration du métier du commercial, aussi bien économique que relationnelle, le manager retrouve une mission d'accompagnement des commerciaux et se fixe comme objectif de les remotiver.

En conclusion, il apparaît que les organisations commerciales sont touchées par la crise dans l'ensemble des secteurs, et que le management commercial répond à cette situation exceptionnelle en accroissant la fréquence des contrôles et en accentuant la pression sur les résultats. Face à la crise, l'ensemble des moyens humains doit être mobilisé vers l'atteinte des objectifs commerciaux car le comportement du client a changé.

\section{Impact de la crise sur le comportement du client}

Lorsque les conseillers bancaires ont été interrogés en mars 2009 sur l'impact de la crise, le premier thème spontanément évoqué par l'ensemble des interviewés a été celui de la modification du comportement des clients. L'ensemble des conseillers aux particuliers a évoqué le besoin de ré assurance et d'explications des clients. La mise en faillite de la banque Lehman Brother en octobre 2008 avait déclenché non seulement des interrogations, mais parfois une véritable panique parmi les clients : «En octobre, les clients étaient paniqués; je recevais une quinzaine de coups de téléphone par jour à ce sujet»; «Il y a eu un effet panique, mais il est maintenant résorbé ». Il a également fallu donner des explications au 
regard de la crise financière et économique : «[Il faut développer] plus de pédagogie [pour expliquer] ce qui se passe au niveau économique ». Les commerciaux étaient dans une démarche plus défensive.

Dans la seconde étude, les commerciaux continuent à percevoir une anxiété chez leurs clients due à la crise et à l'incertitude qui en résulte mais leur réaction commerciale est plus proactive: «Il faut rassurer le client». Cependant, la crise s'étant installée, ce sont des changements plus profonds que mesurent les commerciaux en 2010: «Les rapports avec les clients sont devenus plus tendus»; "Les relations avec les clients se sont durcies». Confrontés à la crise, les clients, ont accru leurs exigences face aux entreprises ; qu'il s'agisse d'acheteurs professionnels ou de particuliers, tous « demandent plus de ristournes», « négocient plus sur le prix». Il s'agit bien d'un accroissement de la capacité de négociation des clients au sens de Porter (1980), la raréfaction de la demande autorisant les clients à faire pression sur leurs fournisseurs. La question se pose alors de savoir comment l'entreprise réagit à cette crise.

\section{Les réactions des commerciaux face à la crise}

Directement confrontés à ces nouveaux comportements des clients, les commerciaux font face à une incertitude et une tension croissante. Selon les équipes, les réactions vont du sursaut collectif à l'exacerbation des rivalités (a) alors même que, d'une façon générale, les commerciaux démontrent leur capacité d'adaptation individuelle (b).

$\underline{\text { a. Réaction à l'incertitude et à la tension : sursaut collectif ou exacerbation des rivalités }}$

La crise s'étant installée dans la durée, les commerciaux en 2010 expriment un stress lié à «l'impossibilité de se projeter dans l'avenir ». Beaucoup insistent : «on est plus stressés »; il existe «des tensions devant les incertitudes du marché ».

Les équipes réagissent aux tensions : 
- $\quad$ soit par la solidarité dans certaines équipes : «On s'entraide » ; « on fait bloc ensemble». "Il y a une consolidation des équipes car nous devons être plus solidaire dans ces moments pour mieux résister à la crise »; «L'équipe s'est ressoudée».

- $\quad$ soit par une compétition exacerbée ailleurs : «l'ambiance est plus tendue »; "l'ambiance s'est dégradée » ; «Il y a plus de compétition entre les commerciaux»; «[Après les licenciements], il ne reste que les meilleurs ».

b. Des capacités d'écoute retrouvées: une démonstration de la capacité d'adaptation du $\underline{\text { commercial }}$

Face aux clients, les commerciaux démontrent des capacités d'adaptation. Dès 2009, on note le développement d'une agressivité commerciale accrue.

«Je vais chez eux et j'essaie de les équiper de manière complète... on est un peu plus agressif commercialement quand on peut le faire ».

« Je fais des ventes plus agressives en disant aux clients qu'ils doivent se dépêcher d'acheter les voitures quand elles sont moins chères »; « Je suis plus incisif avec les clients »; «je suis présent tout le temps sur le terrain »...

En 2010, cette agressivité commerciale est couplée à une vente adaptative au sens de Weitz et al. (1986) : «On s'adapte au client, pas à la crise »; «On est plus à l'écoute et on fait plus de suivi »; «On cherche à fidéliser la clientèle en instaurant un climat de confiance».

Pour certains qui ont travaillé dans les fonctions commerciales depuis de longues années, il s'agit là d'un retour aux bases du métier, les exigences des clients obligeant les commerciaux à retrouver le sens de leur métier : écouter, comprendre les besoins, s'y adapter. 


\section{IV - DISCUSSION DES RESULTATS}

Notre étude indique que les facteurs qui font évoluer le comportement du commercial en période de crise sont à la fois internes (adaptation du commercial) et externes (évolution des comportements des clients et des systèmes de contrôle de l'organisation).

A la question de recherche qui était de savoir si les systèmes de contrôle des forces de vente évoluaient en période de crise, l'analyse que nous avons menée permet de répondre en identifiant à la fois une constance dans les aspects les plus structurants des systèmes de contrôle (e.g. la structure de la rémunération), mais également l'évolution de nombreux éléments plus facilement actionnables par le management de proximité. L'implication renforcée du management de proximité apparaît comme une vérification empirique de la dynamique des systèmes de contrôle suggérée par Darmon et Martin (2011). Face aux conséquences de la crise sur leur marché, les managers de proximité ont accru leur implication dans le pilotage de leur équipe de vente, à la fois en précisant les objectifs de vente dans cette période incertaine, mais aussi en renforçant la rigueur du contrôle exercé sur les agents commerciaux.

Le concept de contrôles informels identifiés par Jaworski (1988) permet d'inclure dans l'analyse l'influence des pairs dans le comportement commercial. Par ailleurs, comme nous l'avons indiqué plus haut, il faut également prendre en compte la capacité d'adaptation propre au commercial, que l'on pourrait associer au «self-control » suggéré par Jaworski (1988), au titre des éléments de contrôle informel. Enfin, la crise a eu un impact significatif sur le comportement du client : dans un marché très disputé, ce dernier a accru son pouvoir de négociation et, dans de nombreux cas, il a modifié sa relation avec le vendeur. La mise en évidence de l'impact de l'évolution du comportement du client peut apparaître comme une 
contribution intéressante de notre étude, en mettant en évidence son rôle dans l'environnement et son influence sur le comportement du commercial, alors que celui-ci n'apparaissait pas dans les cadres développés à ce jour. Ainsi, le nouveau pouvoir de négociation du client peut avoir des conséquences sur le vendeur, en particulier sur la perception qu'il a de son rôle (self control), sa capacité d'adaptation (vente adaptative) ou sa performance.

Nous proposons d'intégrer ce constat dans le cadre théorique développé dans la figure 1 cidessous.

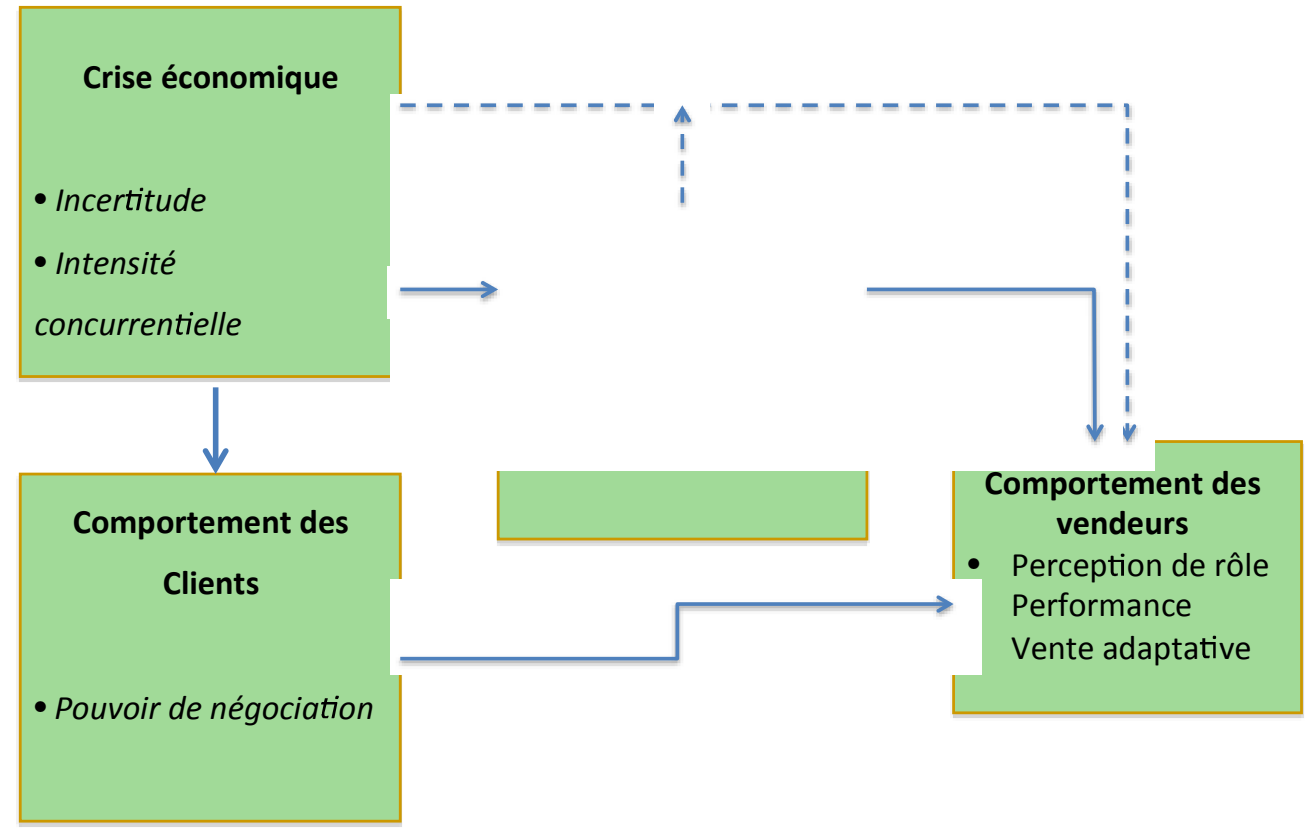

Figure 1. Proposition d'un cadre conceptuel (adapté de Jaworski, 1988)

Nous pouvons formuler deux hypothèses alternatives: tout d'abord nous pouvons envisager une première hypothèse selon laquelle la crise économique a un impact direct sur le comportement du vendeur, en termes de performance, de perception de rôle ou de vente 
adaptative et le système de contrôle intervient alors comme variable modératrice. Ainsi le rôle du management commercial serait de faciliter le travail des commerciaux, de les soutenir et de les accompagner. Une deuxième hypothèse suggérerait que le système de contrôle est une variable médiatrice entre l'influence de l'environnement externe (dans le cas présent, la crise) et le comportement du vendeur. Dans ce cas-là, ce système de contrôle pourrait au contraire accentuer la pression ou le stress causé par la crise. Selon Baron et Kenny (1986), on parle de variable médiatrice lorsque cette dernière permet de rendre compte d'une relation entre une variable indépendante et une variable dépendante. Selon ces mêmes auteurs, la variable médiatrice doit remplir plusieurs conditions : la variable indépendante (la crise) est significativement reliée à la variable médiatrice (systèmes de contrôle); la variable médiatrice est significativement reliée à la variable dépendante (comportement du vendeur); lorsque les liens entre variable indépendante et variable médiatrice, puis variable médiatrice et variable dépendante sont contrôlés, la relation préalablement significative entre variable indépendante (crise) et variable dépendante (comportement du vendeur) devient non significative. On parle alors de médiation complète. Bien entendu, nous ne pouvons que formuler des hypothèses qui devraient être testées par la suite dans une étude quantitative.

Par ailleurs, la crise économique influence directement le comportement du client et modifie la relation créée entre le client et le vendeur : en effet, le client reprenant le pouvoir dans la négociation en modifie les termes. Cette modification de la relation client/vendeur a des conséquences directes sur le comportement du vendeur : tout d'abord le nécessaire retour à la vente adaptative (Weitz et al., 1986) chez le vendeur avec l'adaptation de l'argumentaire et une écoute plus importante du client, etc. De plus, le vendeur est contraint de fournir un effort plus important à toutes les étapes de la vente, qu'il s'agisse de prospection, ou de suivi des clients; en ce sens, nous vérifions la mise en place, dans un contexte de crise, de 
mécanismes individuels de réaction décrits dans d'autres contextes (e.g. Pauchant et Mittroff, 1992). Il s'ensuit une modification du mode de contrôle mis en place par l'entreprise qui doit prendre en compte l'évolution du client et l'adaptation de la négociation par le vendeur : on pourrait ainsi envisager que la relation du système de contrôle de l'entreprise ne sera plus à sens unique vers le vendeur mais que celui par l'intermédiaire du client pourrait également modifier le système de contrôle de l'entreprise.

\section{V - CONCLUSION ET PERSPECTIVES}

L'objectif de cet article était d'étudier l'impact de la crise économique sur les systèmes de contrôle des entreprises et sur le comportement des commerciaux. Nous souhaitions en particulier répondre aux questions suivantes : les systèmes de contrôle ont-ils été modifiés par la crise ? Comment les commerciaux ont-ils ressenti cette évolution et se sont ils adaptés face à leurs clients?

Les résultats de notre étude montrent que l'évolution du management commercial sous l'effet d'une pression accrue se traduit dans l'évolution des systèmes de contrôle de l'entreprise, et particulièrement dans le renforcement du contrôle opéré par le management, un résultat conforme à des études récentes analysant l'accroissement de la pression par les actionnaires (Besson et al., 2008). Nos résultats mettent également en évidence l'importance que la relation vendeur/client peut avoir sur la mise en place des systèmes de contrôle qui prennent en compte le nouveau rôle du client, suggérant ainsi le rôle indirect que les clients jouent dans la mise en place d'un système de contrôle. Cette étude qualitative a ainsi permis de montrer le rôle médiateur du client dans l'impact de la crise économique sur l'entreprise, et plus particulièrement sur l'organisation commerciale et de souligner l'importance du contrôle informel opéré par le commercial lui-même pour répondre à cette évolution (self control). 
Cette étude autorise quelques recommandations managériales: nous suggérons ainsi aux organisations commerciales de «fidéliser dans l'adversité ». C'est en effet dans les premiers temps de la crise, lorsque l'incertitude surgit, qu'il est important pour elles d'apporter des réponses ciblées à leurs clients pour les fidéliser. Dans cette période d'incertitude, le client cherche des repères et apprécie le conseil éclairé d'un établissement financier ou la solution optimisée qui lui est proposée par un fournisseur. Lorsque l'incertitude des débuts de crise s'estompe, le client a construit sa propre réflexion; le cas échéant, il sait quel nouveau pouvoir de négociation il a gagné, et il joue sa carte personnelle. Notre étude a montré que de nombreux commerciaux opèrent d'eux-mêmes un recentrage sur les préoccupations du client, redécouvrent les fondements de la démarche commerciale : écouter le client, le comprendre et lui apporter une solution adaptée. Si ce constat peut sembler une évidence, il apparaît cependant au travers des entretiens menés que certaines organisations ont pu perdre de vue ces fondements, se contentant de gérer des objectifs et des indicateurs de résultats. Pour renforcer les capacités d'adaptation du vendeur, et le soutenir dans un contexte de crise, il est important que l'organisation mobilise des moyens pour lui apporter un appui sur le plan de l'information, du support à la relation client, et du renforcement personnel. En termes d'information le commercial, comme les autres collaborateurs, a besoin de comprendre ce qui se passe, et comment se positionne son entreprise dans la crise. Dans sa relation avec le client, le commercial appréciera un support spécifique : à ce titre, l'exemple de la banque française ayant développé un «kit de crise » pour ses commerciaux est particulièrement emblématique. Enfin, l'accompagnement du management de proximité ne doit pas être perçu par les vendeurs comme un contrôle déresponsabilisant, mais il doit, au contraire, comporter une véritable dimension de renforcement des efforts au sens de Cravens et al. (1993). 
Si nous n'avons pas identifié d'impact en termes de comportements non éthiques lors de nos entretiens, nous ne pouvons pas entièrement préjuger de leur existence, une observation étant mieux à même d'identifier ce type de comportements. En fait, une entreprise confrontée à un contexte de crise et qui a mis en place un système de contrôle basé essentiellement sur les résultats, peut voir les vendeurs développer des comportements non éthiques (Lavorata, 2007). C'est ainsi qu'Ingram et al. (2007) montrent l'importance du «Sales Management Control Strategy » pour développer un comportement éthique du vendeur : pour qu'elle soit efficace, cette stratégie doit intégrer de multiples activités de contrôle et non plus être seulement une opposition entre un contrôle basé sur les résultats et l'autre sur le comportement. Si nous n'avons pas relevé de comportements déviants, nous avons par contre noté une pression généralisée : lorsque celle-ci est perçue comme excessive par les vendeurs, elle peut entraîner une démotivation et un stress accru, et conduire alors au burnout des vendeurs. Celui-ci peut poser un problème pour le management car le burnout peut avoir un effet négatif sur la performance et se traduire par des attitudes négatives vis-à-vis des clients (Hollet et al., 2011).

Notre étude comporte un certain nombre de limites. A ce jour, n'ayant interrogé que des commerciaux terrain, cette étude ne peut rendre compte de la dualité des points de vue qui est pourtant un élément important de compréhension des dynamiques à l'œuvre dans ces périodes d'incertitude économique. Par ailleurs, le cadre conceptuel proposé n'a pas été testé de façon quantitative, et il est possible que d'autres variables puissent être intégrées, comme le conflit de rôle ou bien le climat éthique de l'entreprise. Enfin notre parti pris d'interviewer les commerciaux de terrain ne nous a pas permis de mesurer les conséquences de la crise sur leur performance, et il ne nous est pas possible de formuler une hypothèse à ce sujet. Ces limites 
constituent autant de voies de recherche pour le futur, une première interrogation à lever portant sur la définition d'une performance commerciale en période de crise.

\section{Bibliographie}

Adrot, A., What support does ICT offer to organizational improvisation during crisis response? Unpublished PhD. Dissertation, Robinson College of Business, Georgia State University, 2010.

Anderson E. and Oliver R.L., Perspectives on Behavior-Based Versus Outcome-Based Sales Force Control Systems, Journal of Marketing, vol.51, 1987, p.76-88.

Baldauf, A., Cravens, D.W. et N. F. Pierce, Sales management control research : synthesis and an agenda for future research, Journal of Personal Selling and Sales Management, vol.25, $\mathrm{n}^{\circ} 1,2005$, p.7-26.

Baron R. M. et Kenny D. A., The moderator-mediator variable distinction in social psychological research: Conceptual, strategic and statistical considerations, Journal of Personality and Social Psychology, vol.51, 1986, p.1173-1182.

Barthélémy, J. et Doublet, J-M., Quelques enseignements de la crise, Revue française de Gestion, vol.193,n³, 2009, p. 13-14.

Bergadaà M., Révolution Vente : Enquête sur un changement organisationnel majeur, Village Mondial, Paris, 2000.

Besson, M. Löning, H.; and Mendoza, C., Sticking together under pressure: The risk-sharing approach and trust-building in sales management, European Management Journal, Feb2008, vol.26, n 1 , p. 11-23.

Boddewyn J., A Construct for Comparative Marketing Research, Journal of Marketing Research, vol.3, 1966, p. 149-153.

Challagalla G.N and Shervani T.A., Dimensions and Types of Supervisory Control: Effects on Salesperson Performance and Satisfaction, Journal of Marketing, vol.60, 1996, p. 89-105.

Coombs, W. T., Protecting Organization Reputations During a Crisis: The Development and Application of Situational Crisis Communication, Corporate Reputation Review, vol.3, $\mathrm{n}^{\circ} 10$, Fall 2007, p163-176.

Cravens D.W., Ingram, T.N., LaForge, R.W and Young, C.E., Behavior-based and Outcomebased sales force control systems, Journal of Marketing, vol.57, October 1993, p.47-59.

Darmon R.Y., Le pilotage dynamique de la force de vente, Village mondial, Paris, 2001.

Darmon, R. and Martin, X., A new conceptual framework of sales force control systems, Journal of Personal Selling and Sales management, vol.31, n³, summer 2011, p. 297-310.

Greene W. H., Econometric Analysis, 5th edition, Prentice Hall, 2003.

Hollet-Haudebert S., Mulki J.P. and Fournier, C., Neglected Burnout dimensions: Effect of Depersonalization and personal Non-accomplishment on Organizational Commitment of 
Salespeople, Journal of Personal Selling \& Sales Management, vol.4, n³1, 2011, p. 411428.

Ingram T.N., LaForge R.W. and Schwepker, C.H Jr., Salesperson Ethical Decision Making: The Impact of Sales Leadership and Sales Management Control Strategy, Journal of Personal Selling \& Sales Management, vol.4, n²7, 2007, p. 301-315.

Jaworski B.J., Toward a Theory of Marketing Control: Environmental Context, Control Types, and Consequences, Journal of Marketing, vol.52, July 1988, p.23-39.

Jaworski B. J., Stathakopoulos, V. and Krishnan, H. S., Control Combinations in Marketing: Conceptual Framework and Empirical Evidence, Journal of Marketing, vol.1, n57, 1993, p.57-69.

Kalampalikis N. et Moscovici S., Une approche pragmatique de l'analyse Alceste, Les Cahiers Internationaux de Psychologie Sociale, vol.66, 2005, p. 15-24.

Kouzmin, A., Crisis management in crisis? Administrative Theory \& Praxis, 2, June 2008, p155-183.

Krafft M., An empirical investigation of the antecedents of sales force control systems, Journal of Marketing, vol.63, July 1999, p.120-134.

Lavorata L., Proposition d'une échelle de mesure du climat éthique dans l'entreprise : une application au domaine de la vente en B to B, Recherche et Applications en Marketing, vol.22, n¹, 2007, p.1-24.

Mitroff, II, Pearson, CM and LK Harrington, The essential guide to managing corporate crisis: A step-by-step handbook for surviving major catastrophes. Oxford University Press, 1996.

Ouchi W-G., Markets, Bureaucracies, and Clans, Administrative Science Quarterly, 25(1), 1980, p.129-141.

Oliver, R. and E. Anderson, An Empirical Test of the Consequences of Behavior- and Outcome-Based Sales Control Systems, Journal of Marketing, vol.58, October 1994, p.53-67.

Pauchant, TC and Mittroff, I., Transforming the crisis-prone organization: Preventing individual, organizational, and environmental tragedies. Jossey-Bass, 1992.

Porter, M. Competitive Strategy: Techniques for Analyzing Industries and Competitors, The Free Press, 1980.

Quarantelli, E.L and Dynes, R.R., Response to social crisis and disaster, Annual review of sociology, vol.3, $\mathrm{n}^{\circ} 1,1977$, p. 23-49.

Ramaswami N.S., Marketing controls and dysfunctional employee behaviors: a test of traditional and contingency theory postulates, Journal of Marketing, vol.60, 1996, p.105-120.

Roux-Dufort, C. and Vidaillet, B., The difficulties of improvising in a crisis situation-a case study, International studies of management \& organization, vol.33, $\mathrm{n}^{\circ} 1,2003, \mathrm{p} .86-115$.

Rouziès, D. et Besson, M., Le pilotage des forces de vente : effets pervers des systèmes hybrids, Décisions Marketing, vol.14, mai-août 1998, p. 31-41.

Weick, K.E, Making sense of the organization, Blackwell Publisher, 2001. 
Weitz B. A., Sujan H. and Sujan M., Knowledge, Motivation and Adaptive Behavior: a Framework for improving selling effectiveness, Journal of Marketing, vol.4, $\mathrm{n}^{\circ}$ 50, 1986, p.174-191. 


\section{ANNEXE 1. Guide d'entretien}

\section{Phase d'introduction}

Quelle est votre fonction dans l'entreprise ? Comment est organisée l'entreprise au niveau commercial ?

Comment l'activité commerciale est elle rémunérée ? primes ? objectifs sur résultats? l'aspect qualitatif de votre activité est elle prise en compte (relationnel avec le client? fermeture des comptes ?) ; de quelle manière ? Comment êtes-vous évalué ?

Avez-vous constaté une évolution du management commercial depuis que vous êtes entré dans l'entreprise

\section{Phase de recentrage}

Constatez-vous un impact de la crise dans l'entreprise ? Lequel ? Comment cela se traduit-il ? Y a t'il un impact sur l'organisation commerciale de manière générale ?

Comment la direction commerciale répond elle à la crise ?

Que va-t-il se passer dans les prochaines semaines / mois ?

\section{Phase d'approfondissement}

Cette crise a-t-elle un impact sur votre activité personnelle ?

Sur vos objectifs de vente ? Sur l'évolution de vos missions? Sur votre rémunération?

Constatez-vous un changement dans le climat de l'entreprise? Lequel ? Comment cela se traduit-il ?

Les relations avec votre client ont-elles changé ? De quelle façon ? Se sont elles durcies ?

\section{Phase de conclusion}

Cette crise a-t-elle un impact sur votre comportement commercial ?

Avez-vous modifié votre argumentaire de vente ? De quelle manière ?

Quels sont les produits que vous mettez en avant maintenant et pourquoi ?

\section{Fiche signalétique}

Sexe

Age

Ancienneté dans l'entreprise

Ancienneté dans la fonction commerciale

Localisation 
ANNEXE 2. Composition des deux échantillons

\begin{tabular}{|c|c|c|c|c|c|c|}
\hline ETUDE & Secteur & $\begin{array}{c}\text { Nombre } \\
\text { d'interviewés }\end{array}$ & Femmes & Hommes & $\begin{array}{c}\text { Age } \\
\text { moyen }\end{array}$ & Ancienneté \\
\hline $\begin{array}{c}\text { ETUDE } \\
\text { Mars } \\
\text { 2009 }\end{array}$ & Banque & 12 & 7 & 5 & 33 & 5 \\
\hline $\begin{array}{c}\text { ETUDE } \\
\text { Avril } \\
\mathbf{2 0 1 0}\end{array}$ & & 46 & 14 & 32 & 38 & 10 \\
\hline dont & Immobilier & $(19)$ & $(6)$ & $(13)$ & 35 & 8 \\
\hline & $\begin{array}{c}\text { Bâtiment/ Eq } \\
\text { maison }\end{array}$ & $(8)$ & $(2)$ & $(6)$ & 42 & 7 \\
\hline & Automobile & $(7)$ & $(1)$ & $(6)$ & 40 & 13 \\
\hline & Autres secteurs & $(12)$ & $(5)$ & $(7)$ & 40 & 13 \\
\hline
\end{tabular}

